\title{
Discrete solitons and vortices in the two-dimensional Salerno model with competing nonlinearities
}

\author{
J. Gómez-Gardeñes, ${ }^{1,2}$ B. A. Malomed, ${ }^{3}$ L. M. Floría, ${ }^{1,2}$ and A. R. Bishop ${ }^{4}$ \\ ${ }^{1}$ Departamento de Física de la Materia Condensada and Instituto de Biocomputación y Física de Sistemas Complejos, \\ Universidad de Zaragoza, E-50009 Zaragoza, Spain \\ ${ }^{2}$ Instituto de Ciencia de Materiales de Aragón, Universidad de Zaragoza-CSIC, E-50009 Zaragoza, Spain \\ ${ }^{3}$ Department of Interdisciplinary Studies, School of Electrical Engineering, Faculty of Engineering, \\ University of Tel Aviv, Tel Aviv 69978, Israel \\ ${ }^{4}$ Theoretical Division and Center for Nonlinear Studies, Los Alamos National Laboratory, Los Alamos, New Mexico 87545, USA
}

(Received 8 June 2006; published 13 September 2006)

\begin{abstract}
An anisotropic lattice model in two spatial dimensions, with on-site and intersite cubic nonlinearities (the Salerno model), is introduced, with emphasis on the case in which the intersite nonlinearity is self-defocusing, competing with on-site self-focusing. The model applies, for example, to a dipolar Bose-Einstein condensate trapped in a deep two-dimensional (2D) optical lattice. Soliton families of two kinds are found in the model: ordinary ones and cuspons, with peakons at the border between them. Stability borders for the ordinary solitons are found, while all cuspons (and peakons) are stable. The Vakhitov-Kolokolov criterion does not apply to cuspons, but for the ordinary solitons it correctly identifies the stability limits. In direct simulations, unstable solitons evolve into localized pulsons. Varying the anisotropy parameter, we trace a transition between the solitons in 1D and 2D versions of the model. In the isotropic model, we also construct discrete vortices of two types, on-site and intersite centered (vortex crosses and squares, respectively), and identify their stability regions. In simulations, unstable vortices in the noncompeting model transform into regular solitons, while in the model with the competing nonlinearities they evolve into localized vortical pulsons, which maintain their topological character. Bound states of regular solitons and vortices are constructed too, and their stability is identified.
\end{abstract}

DOI: 10.1103/PhysRevE.74.036607

PACS number(s): 05.45.Yv, 63.20.Ry, 03.75.Lm

\section{INTRODUCTION}

A well-known feature of dynamics in nonlinear lattices is the drastic difference between generic nonintegrable systems, such as the discrete nonlinear Schrödinger (DNLS) equation [1], and exceptional integrable models, such as the Ablowitz-Ladik (AL) equation [2]. While discrete solitons in integrable equations are available in an exact form, their counterparts in nonintegrable systems should be sought numerically [1] or by means of a variational approximation [3], although in specially selected nonintegrable models particular exact soliton solutions can be found too [4].

As the DNLS and AL equations differ in the type of the nonlinear terms (on-site or intersite) and converge to a common continuum limit in the form of the ordinary integrable NLS equation, a combined model can be naturally introduced, mixing the cubic terms of both types. Known as the Salerno model (SM) [5], this combined model is based on the discrete equation

$$
i \frac{d}{d t} \Phi_{n}=-\left(\Phi_{n+1}+\Phi_{n-1}\right)\left(1+\mu\left|\Phi_{n}\right|^{2}\right)-2 \nu\left|\Phi_{n}\right|^{2} \Phi_{n},
$$

where $\Phi_{n}$ is the complex field amplitude at the $n$th site of the lattice, and real coefficients $\mu$ and $\nu$ account for the nonlinearities of the AL and DNLS types, respectively.

The SM was studied in a number of works, see Refs. [6-10] and references therein. It has been demonstrated that Eq. (1) gives rise to static (and, sometimes, moving [7-9]) solitons at all values of the DNLS coefficient, $\nu$, and all positive values of its AL counterpart, $\mu$. Negative values of $\nu$ can be made positive by means of the staggering transfor- mation, $\Phi_{n} \rightarrow(-1)^{n} \Phi_{n}$, and then one may fix $\nu \equiv+1$ by the rescaling, $\Phi_{n} \rightarrow \Phi_{n} / \sqrt{\nu}$ (if $\nu \neq 0$ ). However, the sign of $\mu$ cannot be altered. In particular, there are no solitons in the pure AL model $(\nu=0)$ with $\mu<0$.

The SM with $\mu<0$ is a system with competing nonlinearities, where, in particular, soliton dynamics may be quite different from those in the ordinary SM (with positive $\mu$ ). In the one-dimensional (1D) setting, the SM with $\mu<0$ was introduced in Ref. [10]. It is relevant to mention that, while the SM was originally proposed as a dynamical model in a rather abstract context, the direct physical realization, has recently been found as a limit form of the Gross-Pitaevskii equation for a Bose-Einstein condensate (BEC) of atoms with magnetic momentum (actually, ${ }^{52} \mathrm{Cr}$ [11]) trapped in a deep optical lattice [12]. In that case, the on-site nonlinearity accounts (as usual) for collisions between atoms, while the intersite nonlinear terms take into account long-range dipoledipole interactions, which may be attractive $(\mu>0)$ or repulsive $(\mu<0)$, if the external magnetic field aligns the atomic momenta, respectively, parallel to the lattice or perpendicular to it.

Soliton solutions of the SM with $\mu<0$ were also investigated in Ref. [10]. It was demonstrated that Eq. (1) with $\mu$ $<0$ and normalization $\nu=1$ gives rise to soliton families of two different types, viz., ordinary ones (which are similar to solitons in the SM with $\mu>0$ ) and cuspons, which are characterized by a higher curvature in the center than in the exponentially decaying tails. At the border between the two species of soliton solutions, a special soliton appears, in the form of a peakon. In the continuum limit of Ref. [10] with $\mu<0$, a peakon solution is available in an exact analytical 
form, while cuspons do not exist in this limit. Stability of the discrete solitons in the SM with the competing nonlinearities was also investigated in Ref. [10], with a conclusion that only a small subfamily of the ordinary solitons is unstable, and all cuspons, including the peakon, are stable. In fact, the stability and instability of the ordinary solitons were correctly predicted by the known Vakhitov-Kolokolov (VK) criterion [13] (its definition is given below), while it does not apply to cuspons.

Bound states of solitons with phase difference $\Delta \varphi=0$ and $\pi$ were also studied in Ref. [10]. For ordinary solitons, the character of the bound states is the same as in the DNLS equation, i.e., stable and unstable bound states have, respectively, $\Delta \varphi=\pi$ and 0 . However, they exchange the stability precisely at the point where the bound solitons are peakons, stable bound states of cuspons having $\Delta \varphi=0$.

Moving quasisoliton states, composed of a moving localized core and a spatially extended background, were also found in Ref. [10], up to a critical value of the strength of the self-defocusing intersite nonlinearity, $|\mu|$. It was demonstrated that collisions between moving solitons always lead to their merger into a single pulse.

The SM can be introduced in the two-dimensional (2D) setting as well. This may find a straightforward physical application as a discrete model for the BEC of dipolar atoms trapped in a deep two-dimensional optical lattice, cf. Ref. [12]; in that case, assuming that a strong magnetic field aligns the momenta perpendicular to the lattice plane (and the condensate is strongly confined in the vertical direction), one will again deal with the dipole-dipole repulsion, i.e., $\mu<0$ in Eq. (2), see below.

New dynamical properties featured by solitons in the 1D SM with competing nonlinearities suggest investigating solitons in the 2D version of the model, which is the subject of the present work. We aim to consider the 2D lattice model in its general anisotropic form, which will make it possible to trace a transition between the 1D and 2D versions of the SM. After setting up the 2D model in Sec. II, we present soliton families and their stability in Sec. III. Similar to the 1D case, both ordinary solitons and cuspons are found in the 2D model, with peakons precisely at the border between them. The stability analysis is performed through the computation of the Floquet eigenvalues for small perturbations, and verified in direct simulations. As a result, we identify stability diagrams in relevant parameter planes. Unstable solitons of the ordinary type are not destroyed, but are transformed into pulsons (breathing solitons), while all cuspons are found to be stable. The stability of bound states of solitons is also analyzed.

A new class of solitons, possible in the 2D model, are discrete vortices [15]. We investigate vortex solitons of two types, crosses and squares, in Sec. IV (in the framework of the isotropic model). Analysis of small perturbations reveals parametric stability regions (which turn out to be rather narrow) for the vortices, and helps to identify various bifurcations (including a generic Hamiltonian Hopf bifurcation) responsible for their destabilization. Direct simulations demonstrate that the instability transforms the vortices into ordinary solitons in the case of $\mu>0$, and into vortex pulsons, that keep the vortical topology, in the most interesting case of competing nonlinearities, $\mu<0$. Finally, we introduce bound states of vortex crosses and analyze their stability.

\section{THE MODEL}

The 2D version of the Salerno model is based on the equation for complex lattice variables $\Phi_{n, m}(t)$,

$$
\begin{aligned}
i \frac{d}{d t} \Phi_{n, m}= & -\alpha\left[\left(\Phi_{n+1, m}+\Phi_{n-1, m}\right)+C\left(\Phi_{n, m+1}+\Phi_{n, m-1}\right)\right] \\
& \times\left(1+\mu\left|\Phi_{n, m}\right|^{2}\right)-2 \nu\left|\Phi_{n, m}\right|^{2} \Phi_{n, m},
\end{aligned}
$$

where $\alpha$ is the strength of the coupling between adjacent lattice sites [in Eq. (1), $\alpha \equiv 1$ ], and $C$ accounts for anisotropy of the $2 \mathrm{D}$ lattice $[C=1$ and 0 correspond, respectively, to the isotropic 2D lattice and its 1D counterpart, see Eq. (1)]. Variation of $C$ will make it possible to observe the dimensionality crossover (from 1D to 2D). The nonlinear coefficients in Eq. (2), $\nu$ and $\mu$, have the same meaning as in the 1D model.

Similar to the 1D version of the SM, Eq. (2) conserves the Hamiltonian and norm,

$$
\begin{gathered}
\mathcal{H}=-\alpha \sum_{n, m}\left(\Phi_{n, m} \bar{\Phi}_{n+1, m}+\Phi_{n+1, m} \bar{\Phi}_{n, m}\right)-\alpha C \sum_{n, m}\left(\Phi_{n, m} \bar{\Phi}_{n, m+1}\right. \\
\left.+\Phi_{n, m+1} \bar{\Phi}_{n, m}\right)-\sum_{n, m}\left[\frac{2 \nu}{\mu}\left|\Phi_{n, m}\right|^{2}-\frac{2 \nu}{\mu^{2}} \ln \left(\left.|1+\mu| \Phi_{n, m}\right|^{2}\right)\right] \\
\mathcal{N}=\frac{1}{\mu} \sum_{m, n} \ln \left(\left.|1+\mu| \Phi_{n, m}\right|^{2} \mid\right) .
\end{gathered}
$$

It is also relevant to take a continuum limit of Eq. (2). To this end, we adopt the normalization $\alpha=\nu=1$ (see below), proceed from the discrete coordinates $(n, m)$ to continuous ones, $(x, y) \equiv(n / \sqrt{\alpha}, m / \sqrt{C \alpha})$, and define $\Phi_{m, n}$ $\equiv(1 / \sqrt{\alpha}) \Psi(x, y) \exp [2 i \alpha(1+C) t]$. Then, the continuum counterpart of Eq. (2) is

$$
i \Psi_{t}+\left(1+\mu|\Psi|^{2}\right)\left(\Psi_{x x}+\Psi_{y y}\right)+2[(1+C) \mu+1]|\Psi|^{2} \Psi=0 .
$$

[Note that this equation may always be cast in the isotropic form.] Stationary solutions to Eq. (5), $\Psi(r, t)=e^{-i \omega t} F(r)$, where $r \equiv \sqrt{x^{2}+y^{2}}$, can be found in an exact form for $\omega$ $=2\left[(1+C)-|\mu|^{-1}\right]$. At this value of the frequency, Eq. (5) reduces to the linear equation, $F^{\prime \prime}+r^{-1} F^{\prime}+\omega F=0$, whose solution is available in terms of the Bessel functions (however, this solution cannot be simultaneously localized and nonsingular). For $\mu<0$, the amplitude of the solutions is limited to $F(0) \leq|\mu|^{-1 / 2}$, the limiting case of $F(0)=|\mu|^{-1 / 2}$ corresponding to a radial peakon, with the asymptotic form $F(r)$ $=|\mu|^{-1 / 2}-c r+O\left(r^{2}\right)$ at $r \rightarrow 0$, where the constant $c$ is related to $\omega$ and $\mu: c^{2}=|\mu|^{-1}-(1+C)+\omega / 2$. However, the peakon solution and the value of $\omega$ corresponding to it (at given $\mu<0$ ) cannot be found in an exact form, unlike the 1D version of the model [10]. Finally, we notice that in both cases 

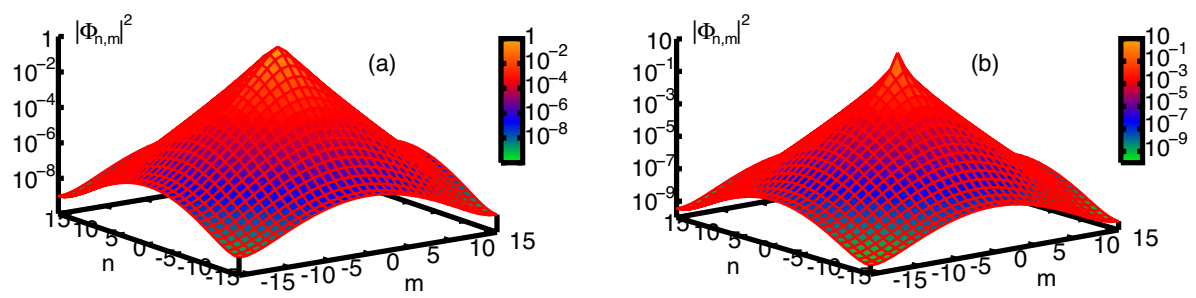
of positive and negative $\mu$, the dispersive nonlinear term in Eq. (5), $\mu|\Psi|^{2}\left(\Psi_{x x}+\Psi_{y y}\right)$, prevents collapse. Therefore, quasicollapse [16] is not expected in the discrete model either.

\section{DISCRETE SOLITONS}

\section{A. Soliton families}

First, we focus on solutions to Eq. (2) in the form of pinned discrete solitons. For this purpose, we aim to construct families of the soliton solutions (labeled by their internal frequency $\omega_{b}$ ) for different values of $\mu$ and $C$, keeping $\nu \equiv 1$ and $\alpha \equiv 1$, which is possible due to the scaling invariance of the model. We compute the solutions by means of a numerical-continuation procedure based on a shooting method (which makes use of the Newton-Raphson scheme). We start from the well-known soliton solutions for the 2D DNLS equation, corresponding to $\mu=0$, which, in turn, can be obtained by the continuation in $\alpha$ from the anticontinuum limit, $\alpha=0$, up to the above-mentioned fixed value, $\alpha \equiv 1$. Then, our continuation seeds a set of solutions at different frequencies and several different values of $C$, which are subsequently continued in $\mu$. Typically, the difference between the soliton frequencies used for each $C$ was $\delta \omega_{b}=8 \times 10^{-2}$ (which is also the lowest frequency taken close to the edge of the phonon band), and the continuation step in $\mu$ was $\delta \mu$ $=2 \times 10^{-2}$. In this way, we scanned the family of discrete solitons in the entire $\left(C, \mu, \omega_{b}\right)$ space. The $2 \mathrm{D}$ lattice used in the computations was typically an $N \times N$ square with periodic boundary conditions. We used different lengths $N$, depending on the width of the soliton (so as to have $N$ much larger than the size of the soliton, or of soliton bound states, if the latter were studied).

As noted above, varying $C$ from 0 to 1 allows one to observe the transition from a one- to a two-dimensional lattice. We will concentrate on the existence of 2D cuspons and their stability. In the $1 \mathrm{D}$ case, cuspons exist for $\mu<0$, when the on-site and intersite nonlinearities are competing [10]. These solutions present highly localized profiles, with the decay rate around the localization center higher than in tails of the solutions. Another relevant result of the 1D case is that low-frequency solitons are unstable in a narrow interval of negative values of $\mu$. The instability observed in the 1D case transforms the discrete soliton into a pulsonic state. Note that the standard 2D DNLS lattice features a similar unstable behavior for low-frequency solitons, and a similar instability was previously also found in the ordinary $(\mu>0)$ 2D SM $[17,18]$. It is interesting to find a link of the soliton instability in the 2D SM (2) with $\mu>0$ and the above-mentioned instability of solitons in the $1 \mathrm{D}$ model with $\mu<0$, which we will do in this paper (see below).
FIG. 1. (Color online) Intensity profiles, $\left|\Phi_{n, m}\right|^{2}$, of two discrete solitons found for $C=1$ (isotropic case) and frequency $\omega_{b}$ $=4.22$ : (a) $\mu=-0.2$; (b) $\mu=-0.88$. The latter solution is a cuspon, which features stronger localization at its center than in the tails.
First, we consider the shape of solutions produced by the tive and of sufficiently large absolute value. In Fig. 1, we display discrete solitons found at $C=1$ (in the isotropic lattice) and $\omega_{b}=4.22$, for two different values of $\mu$. As seen in Fig. 1(b), the cuspon indeed features a higher spatial decay rate around its center, $\left(n_{0}, m_{0}\right)$, than far from it.

To characterize the transition from usual discrete solitons to the cuspons, we fitted the decaying tail of the soliton along the vertical and horizontal directions on the lattice, $\left(n_{0}, m \rightarrow \pm \infty\right)$ and $\left(n \rightarrow \pm \infty, m_{0}\right)$, to the expected asymptotic forms, $\left|\Phi_{n_{0}, m \rightarrow \pm \infty}^{(\text {asymp })}\right|=A_{y} \exp \left(-\Gamma_{y}\left|m-m_{0}\right|\right)$ and $\left|\Phi_{n \rightarrow \pm \infty, m_{0}}^{\text {(asymp) }}\right|=A_{x} \exp \left(-\Gamma_{x}\left|n-n_{0}\right|\right)$, respectively. Once two pairs of parameters $\left(A_{x}, \Gamma_{x}\right)$ and $\left(A_{y}, \Gamma_{y}\right)$ are found, one can determine whether the decay rate (localization degree) around the soliton's center is higher or lower than in the tails, by defining two sharpness parameters (cf. a similar definition adopted in Ref. [10] for the 1D case), $\gamma_{x} \equiv A_{x}-\left|\Phi_{n_{0}, m_{0}}\right|$, and $\gamma_{y} \equiv A_{y}-\left|\Phi_{n_{0}, m_{0}}\right|$. Obviously, $\gamma_{x} \equiv \gamma_{y}$ in the isotropic model $(C=1)$; however, $\gamma_{x}$ and $\gamma_{y}$ are different in anisotropic lattices. We have computed both quantities as the continuation in $\mu$ was performed for each soliton at frequency $\omega_{b}$. For a given pair of parameters $C$ and $\omega_{b}$, it was found that, for higher (in particular, less negative) values of $\mu$, both $\gamma_{x}$ and $\gamma_{y}$ are positive, thus pointing out that the localized states are ordinary discrete solitons (not cuspons). Decreasing $\mu$, one finds a critical value, $\mu=\mu_{y}$, at which $\gamma_{y}=0$, which corresponds to a peakon profile [10] in the (vertical) direction of weak coupling, $\left(n_{0}, m\right)$. If $\mu$ is further decreased, we then have $\gamma_{y}<0$, while $\gamma_{x}$ is still positive (i.e., the soliton is a semicuspon), until the second critical point is reached, $\mu=\mu_{x}<\mu_{y}$, where $\gamma_{x}=0$, and the soliton assumes a peakon profile in the (horizontal) direction of strong coupling, $\left(n, m_{0}\right)$. Finally, at $\mu<\mu_{x}$, both $\gamma_{x}$ and $\gamma_{y}$ are negative, and the discrete soliton is a cuspon in both directions. Figure 2 shows the critical values, $\mu_{x}$ and $\mu_{y}$, versus $\omega_{b}$ for several fixed values of $C$. As noted above, $\mu_{x}=\mu_{y}$ when $C=1$, while for $C=0$ (the 1D limit), only $\mu_{x}$ exists.

\section{B. Linear stability analysis for discrete solitons}

Concomitant with the continuation of the soliton solutions in $\mu$, we examined their linear stability. To this end, we constructed the Floquet operator, $\hat{\mathcal{F}}$, that governs the transformation of a small perturbation, $\delta \Phi$, on top of the stationary solution after period $T_{b} \equiv 2 \pi / \omega_{b}, \delta \Phi\left(t_{0}+T_{b}\right)=\hat{\mathcal{F}} \delta \Phi\left(t_{0}\right)$.

To build the operator, we make use, as usual, of the linearization of Eq. (2). By computing eigenvalues of operator $\hat{\mathcal{F}}$ (Floquet multipliers),$\lambda_{j}(j=1, \ldots, N \times N)$, and the respec- 


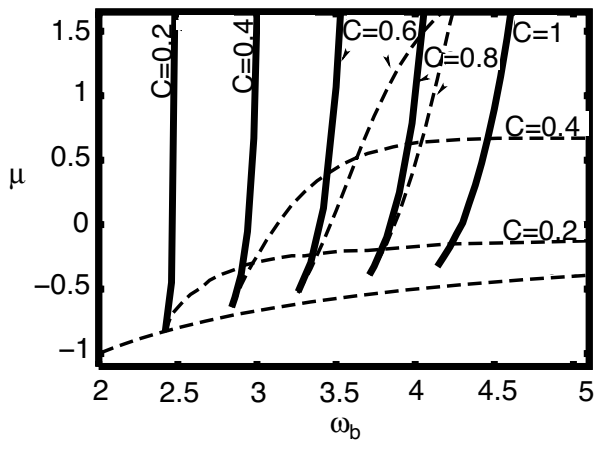

FIG. 2. Solid and dashed curves show, respectively, the critical values of the intersite nonlinearity coefficient, $\mu_{y}$ and $\mu_{x}$, at which $\gamma_{y}=0$ and $\gamma_{x}=0$, as a function of the soliton's frequency for several values of the anisotropy parameter $C$.

tive eigenvectors, one can determine the stability of the discrete solitons and identify unstable perturbation modes (if any). Due to the symplectic (conservative) character of the model, the only possibility for the stability is $\left|\lambda_{j}\right|=1$ for all $j$. Performing the Floquet analysis for every computed solution, we have generated a full stability diagram in the $\left(\omega_{b}, \mu\right)$ plane (including both positive and negative $\mu$ ) for several values of $C$. This is shown in Fig. 3. For $\mu>0$ we observe, as expected (see $[17,18]$ ), an unstable region corresponding to the low-frequency solitons. In this case, the instability development leads to a pulsonic state, similar to what was found in Ref. [10] in the 1D version of the model. On the other hand, for $\mu<0$, all cuspons are found to be linearly stable. This is a new result for 2D lattices of the DNLS type.

Another important result concerns the relation to instability of $1 \mathrm{D}$ solutions at $\mu<0$. Taking a close look at the evolution of the stability diagrams as $C$ decreases, one can monitor a transition from the $2 \mathrm{D}$ isotropic model to its $1 \mathrm{D}$ counterpart. We thus observe (see contour plots in Fig. 3) that there is a subregion in the $\left(\omega_{b}, \mu\right)$ plane, for each value of $C$, where the largest values of $\left|\lambda_{j}\right|$ are much higher than in the rest of the unstable region. This subregion continuously deforms as $C$ varies, and, as $C \rightarrow 0$, it approaches the unstable region found in the 1D model [10].

We have also checked the validity of the VakhitovKolokolov (VK) criterion for the stability of solitons in the present model. This criterion states that a necessary condition for the soliton's stability is $\partial \mathcal{N} / \partial \omega_{b}>0$ [13]. We have computed the norm of the solutions as per Eq. (4), to generate surfaces $\mathcal{N}\left(\omega_{b}, \mu\right)$ for several values of $C$, see Fig. 3. In the figure, we have also plotted curves at which $\partial \mathcal{N} / \partial \omega_{b}$ changes sign, thus separating the predicted stable and unstable regions. Comparison with the rigorous results produced by the Floquet analysis confirms the validity of the VK criterion. A noteworthy feature of surfaces $\mathcal{N}\left(\omega_{b}, \mu\right)$ is the divergence when the soliton's amplitude attains the value $\left|\Phi_{n_{0}, m_{0}}\right|^{2}=1 /|\mu|$. In the 1D model $(C=0)$, this happens for an exact peakon solution, whereas for $C>0$ we observe that the divergence curve in the $\left(\omega_{b}, \mu\right)$ plane is located below the curve of $\mu=\mu_{y}\left(\omega_{b}\right)$, i.e., it happens for cuspon states. Examining the norm for cuspons with the amplitude exceeding $1 / \sqrt{|\mu|}$, we conclude that $\partial \mathcal{N} / \partial \omega_{b}<0$ for all $\omega_{b}$. In this region (after the divergence of the norm occurs), the VK criterion predicts the instability of cuspon states contrary to the results of the Floquet analysis. [The stability of perturbed cuspons was also confirmed by direct simulations of Eq. (2).] Hence, the VK criterion does not apply to the cuspons with $\left|\Phi_{n_{0}, m_{0}}\right|>1 / \sqrt{|\mu|}$.

\section{Bound states of discrete solitons}

In addition to isolated solitons in Eq. (2), we studied numerically their bound states. Two types, in-phase and $\pi$-out-of-phase, of pairs of identical solitons, with the same

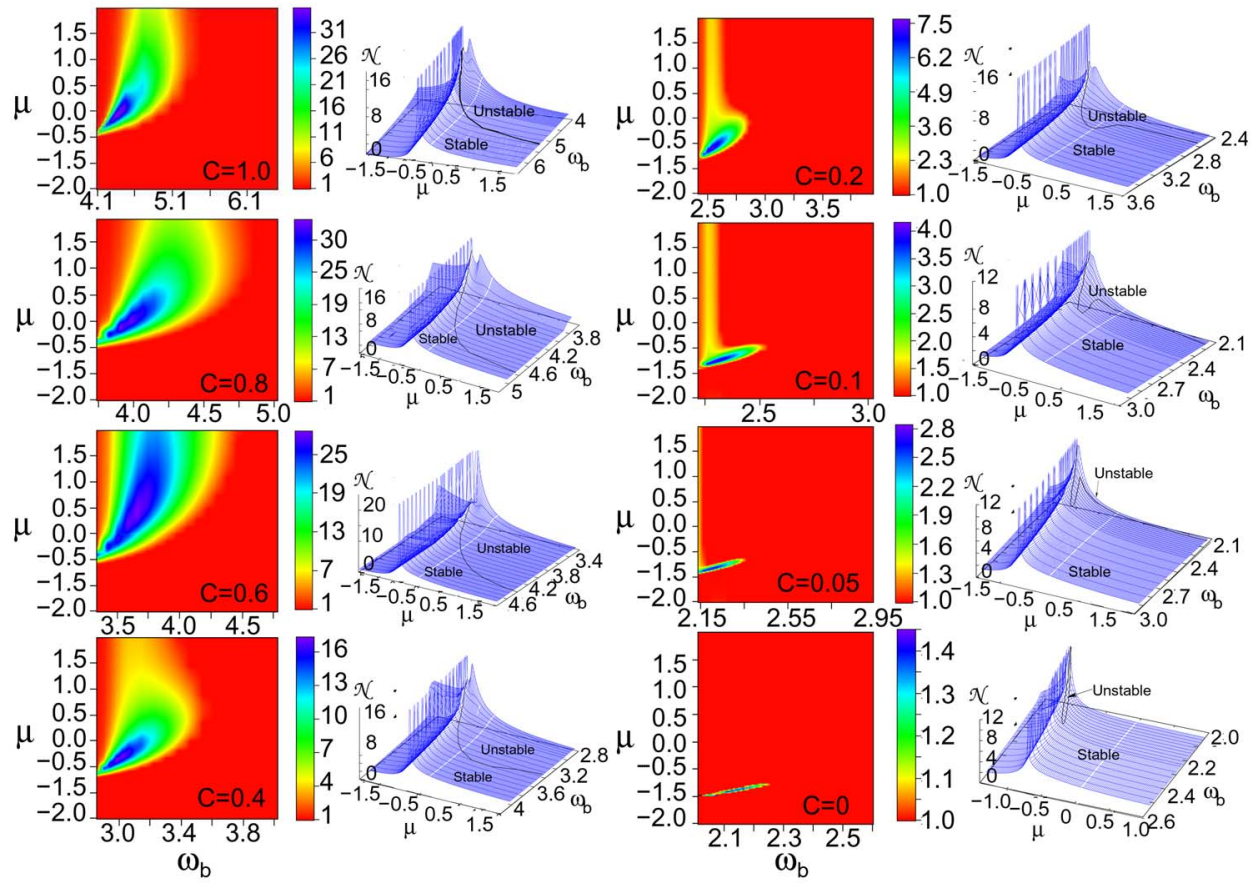

FIG. 3. (Color online) Stability diagrams in the $\left(\mu, \omega_{b}\right)$ plane corresponding to different values of the anisotropy parameter $C$. Contour plots show maximum absolute values of the Floquet multipliers, $\max \left\{\left|\lambda_{j}\right|\right\}$. Beside each contour plot, norm surfaces, $N\left(\mu, \omega_{b}\right)$, are plotted for the corresponding values of $C$. Curves at which the sign of slope $\partial \mathcal{N} / \partial \omega_{b}$ changes are plotted on top of the surfaces. According to the Vakhitov-Kolokolov criterion, these curves separate stability and instability regions. 


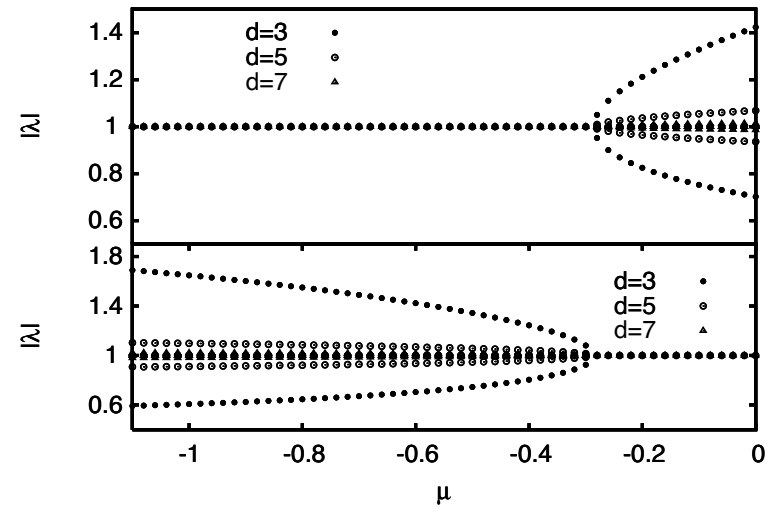

FIG. 4. The absolute value of the Floquet multipliers as a function of $\mu$ for in-phase (top) and out-of-phase (bottom) axis-aligned bound states of solitons with $\omega_{b}=7$. The figure shows cases when the two soliton centers are separated by $d=3,5$, and 7 . It can be observed that, irrespective of the value of $d$, the stability interchange occurs at $\mu=-0.3$.

frequency $\omega_{b}$ and different distances between them, were analyzed. For this purpose, we first continued these solutions, at $\mu=0$, from the anticontinuum limit up to the $2 \mathrm{D}$ DNLS equation $(C=\nu=\alpha=1)$, and then decreased the value of $\mu$ into the region of competing nonlinearities $(\mu<0)$. At the same time, the linear stability analysis of these periodic solutions was performed by the numerical computation of their Floquet spectra.

We have computed two different patterns of bound states of solitons. The first type consists of two discrete solitons with their centers, $\left(n_{0}^{(j)}, m_{0}^{(j)}\right), j=1,2$, lying on the same lattice axis (so that $n_{0}^{(1)}=n_{0}^{(2)}$ or $m_{0}^{(1)}=m_{0}^{(2)}$ ), whereas for the second type of bound states the centers are related by $n_{0}^{(1)}=n_{0}^{(2)} \pm d$ and $m_{0}^{(1)}=m_{0}^{(2)} \mp d$, i.e., they are aligned along a diagonal of the lattice. In Fig. 4, we show the absolute value of the Floquet multipliers as a function of $\mu$ for in-phase and out-of-phase bound states, aligned along a lattice axis for the case of $\omega_{b}=7.0$, with three different values of the distance between soliton centers in the pair. Results of similar computations for the diagonal-aligned bound states with $\omega_{b}=8.0$ are shown in Fig. 5. As in the 1D version of the model [10], for $\mu=0$, in-phase bound states are linearly unstable (the more unstable the closer solitons are in the pair), while out-of-phase pairs are stable. As observed in Figs. 4 and 5 , at $\mu=-0.3$ for the pattern of the first type $\left(\omega_{b}=7.0\right)$, and at $\mu=-0.25$ for the second one $\left(\omega_{b}=8.0\right)$, the in-phase bound states become stable regardless of the distance between solitons. Simultaneously, out-of-phase states become unstable, also regardless of the separation between soliton centers. The same stability exchange between in- and out-ofphase states was observed in the 1D case [10], where it occurs at the value of $\mu$ at which the discrete soliton solution is a peakon. However, in the $2 \mathrm{D}$ case the solitons in the pair are cuspons on both sides of the stability-exchange point. Nevertheless, we find, for both types of the bound states, that the values of $\mu$ at this point are exactly the same as those at which the cuspon's norm, $\mathcal{N}\left(\omega_{b}, \mu\right)$, diverges (see the previous subsection). In other words, the stability interchange between in- and out-of-phase bound states is associated with

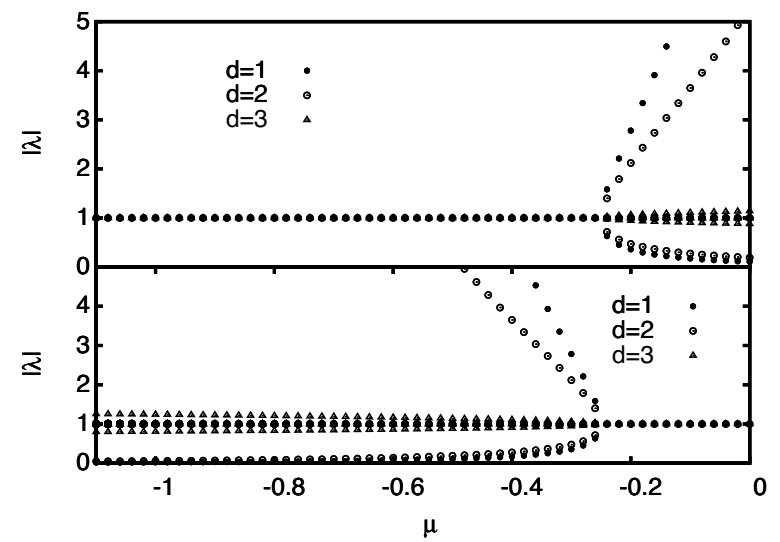

FIG. 5. The same as in the previous figure for in-phase (top) and out-of-phase (bottom) diagonal-aligned bound states of solitons with $\omega_{b}=8$. Shown are the results for the states with the separation between the soliton centers $d=1,2$, and 3 . It can be observed that all these states undergo the stability exchange at $\mu=-0.25$.

the divergence of the soliton norm $\mathcal{N}\left(\omega_{b}, \mu\right)$, rather than to the appearance of a peakon (contrary to the $1 \mathrm{D}$ case, where the emergence of a peakon and norm divergence occurs simultaneously). Thus, although the divergence of the norm does not switch the stability of single discrete solitons, it marks the stability border of bound states of the solitons, regardless of their size and orientation relative to the lattice.

\section{DISCRETE VORTICES}

A natural generalization of the fundamental discrete solitons is discrete vortices, which are well-known solutions of the ordinary 2D DNLS model [15]. A vortex is characterized by the phase circulation around its center, $\Delta \theta$, that must be a multiple of $2 \pi$. Hence they may be labeled by an integer number (vorticity, or topological charge), $S \equiv \Delta \theta /(2 \pi)$.

In this work, we consider vortices only in the isotropic model $(C=1)$, with the objective of analyzing the effects of competing nonlinearities on them. (In the framework of the 2D DNLS model, the influence of the lattice anisotropy on fundamental and vortical discrete solitons was studied in Ref. [19].) We will construct two types of vortices, on-siteand off-site-centered ones (alias vortex crosses and vortex squares), both with $|S|=1$. Vortex squares are characterized by the number of lattice bonds, $M$, that each side of the square comprises; in this work, we only deal with $M=1$. Two examples of these two species of the solutions are plotted in Fig. 6.

\section{A. Vortex crosses}

To construct fundamental $(|S|=1)$ vortex crosses centered around the lattice site $\left(n_{0}, m_{0}\right)$, we start with the anticontinuum $(\alpha=0)$ DNLS $(\mu=0)$ limit. The corresponding seed pattern includes nonzero fields

$$
\Phi_{n_{0}, m_{0}+1}=-i \Phi_{n_{0}+1, m_{0}}=-\Phi_{n_{0}, m_{0}-1}=i \Phi_{n_{0}-1, m_{0}}=\sqrt{\omega_{b} / 2} .
$$

Then, by adiabatically increasing the intersite coupling (Newton continuation in $\alpha$ ), we reach the isotropic DNLS 

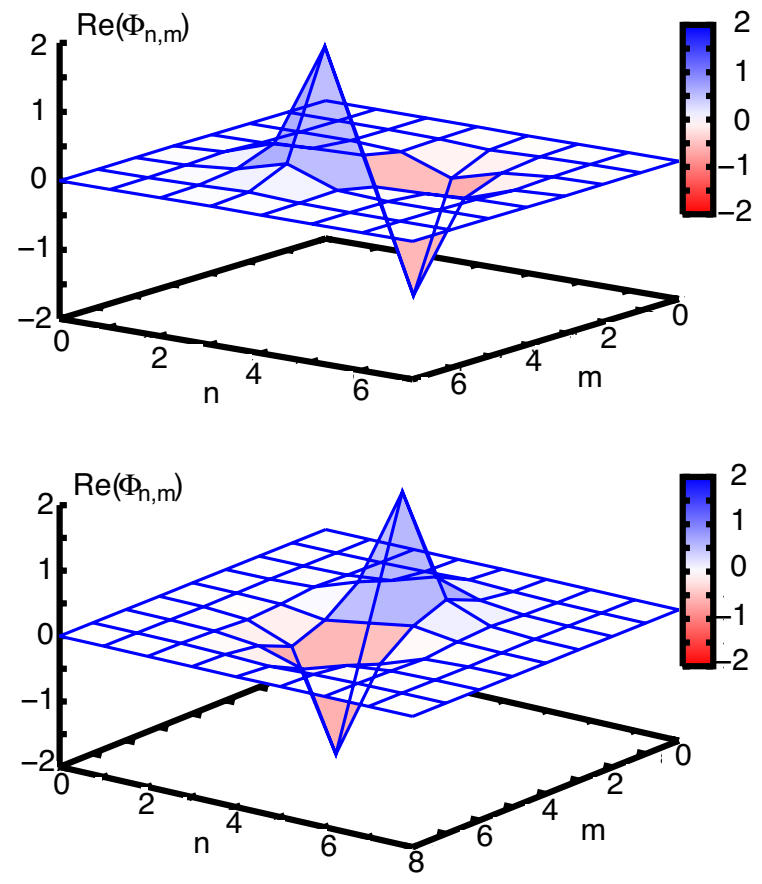

FIG. 6. (Color online) Two examples of fundamental $(|S|=1)$ discrete vortices. Profiles of the real part of the square vortex with $M=1$ and vortex cross are shown in the top and bottom panels, respectively. Both solutions are found for $\mu=-0.4$ and $\omega_{b}=7.0$ (as noted in the text, we fix $C=\alpha=1$ for the vortex solutions).

model, and start the continuation to positive values of the intersite nonlinearity, $\mu$. Performing the continuation in $\alpha$ at $\mu=0$, we have found that, for low-frequency vortex solutions, there is a critical value, $\alpha_{c}$, that depends on frequency $\omega_{b}$, at which a Hamiltonian Hopf bifurcation (HHB) [20] occurs, the vortex solution being unstable at $\alpha>\alpha_{c}\left(\omega_{b}\right)$. This phenomenon was already reported in previous works $[15,19]$.

Higher-frequency vortex solutions, which are stable in the DNLS equation in the considered range of parameters, undergo destabilization through a bifurcation of the same type as a result of the continuation in $\mu$, at $\alpha=1$. The Hamiltonian-Hopf character of the bifurcation can be seen in Fig. 7(c), which shows the Floquet spectrum after the bifurcation: it is seen that a quadruplet of complex eigenvalues $\lambda_{j}$ exit the unit circle. After this (first) bifurcation, further bifurcations of the same type occur at increasing values of $\mu$, as observed in the right part of Fig. 7(a). Similar to what was reported in Ref. [15] for the DNLS model, in direct simulations unstable vortex crosses evolve into on-site-centered fundamental discrete solitons (with $S=0$ ) by transferring almost all the energy to one of the sites that originally formed the cross. The corresponding instability border (for $\alpha=1$ ) in the $\left(\mu, \omega_{b}\right)$ plane is depicted by the right curve of Fig. 7(b).

More interesting is the case of $\mu<0$. In this regime, we have found that fundamental vortex crosses experience another bifurcation, with a quadruplet of Floquet eigenvalues leaving the unit circle at $\lambda=+1$ (the so-called harmonic bifurcation). With the decrease of $\mu$, the corresponding two pairs of eigenvalues move along the real axis in the opposite direction, until each pair breaks up, as shown in Fig. 7(d). The unstable eigenvectors, $\delta \Phi^{*}$ and $\delta \Phi^{* *}$, associated with
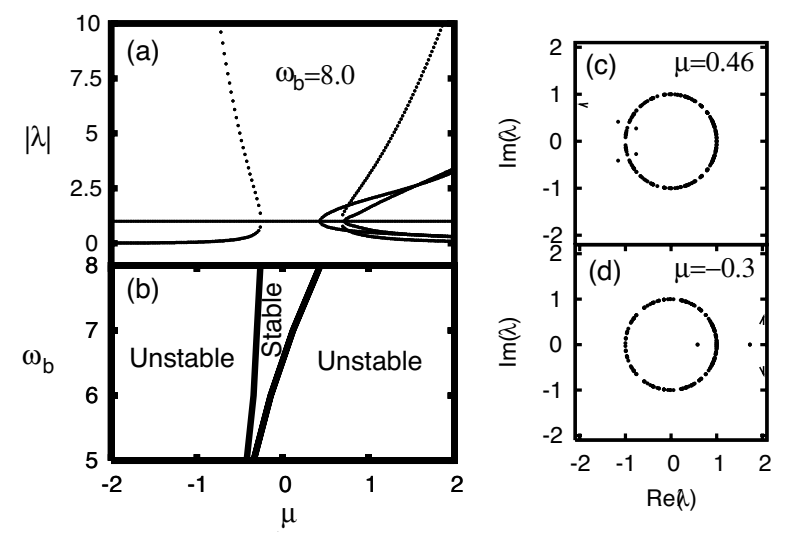

FIG. 7. (a) The absolute value of the Floquet multipliers as a function of $\mu$ for a vortex cross with $\omega_{b}=8$. Two bifurcations can be inferred from the Floquet distributions in panels (c) and (d): a Hamiltonian Hopf bifurcation at $\mu=0.46$, and a harmonic bifurcation (which is defined in the text) at $\mu=-0.3$. A similar set of two bifurcations is found at other frequencies. The entire stability diagram is displayed in panel (b), showing a narrow stability region.

this bifurcation are plotted in Figs. 8(a) and 8(b) (in this notation, * does not stand for complex conjugation). The shape of each eigenvector reveals strong localization at two opposite sites of the vortex cross, each one separately breaking the spatial symmetry (2D isotropy) of the original solution. Adding a small perturbation to the solution along one unstable direction causes oscillations of the amplitudes around the vortex' center, as shown in Fig. 8(c). Such behavior persists at longer times; in fact, the vortex pattern does not disappear but rather suffers irregular modulations of its local amplitudes.

This picture of the instability development supplements the stability diagram for the fundamental vortex crosses, which is displayed in Fig. $7(\mathrm{~b})$ in the $\left(\mu, \omega_{b}\right)$ plane (as noted above, for the isotropic model, with $C=1$ ). Note that the border of the instability that transforms the vortex cross into its oscillatory counterpart (the left curve in the figure) stays in the $\mu<0$ region, even for large frequencies. Therefore, unlike the HHB described above, this instability is dominated by the competition between the defocusing intersite and focusing on-site nonlinearities. A further insight into the nature of this bifurcation is provided by the observation that it coincides exactly with the divergence of norm $\mathcal{N}\left(\omega_{b}, \mu\right)$ of the discrete soliton (and of the vortex cross solution), and thus it coincides with the stability interchange between inphase and out-of-phase bound states analyzed above in Sec. III C.

Regarding the vortex cross as made up of two (perpendicular) out-of-phase bound states of solitons (say, left-right and top-bottom), one would be tempted to interpret the quadruplet of eigenvalues leaving the unit circle at +1 as the two pairs of eigenvalues that signal the simultaneous instability of both out-of-phase bound states. At least, this interpretation would explain the fact that a quadruplet of eigenvalues simultaneously leave the unit circle at +1 , and it is fully consistent with the shape of the Floquet eigenvectors in Fig. 8. This interpretation suggests that the bifurcation of vortex crosses occurring in the left part of Fig. 7(b) is the same one 


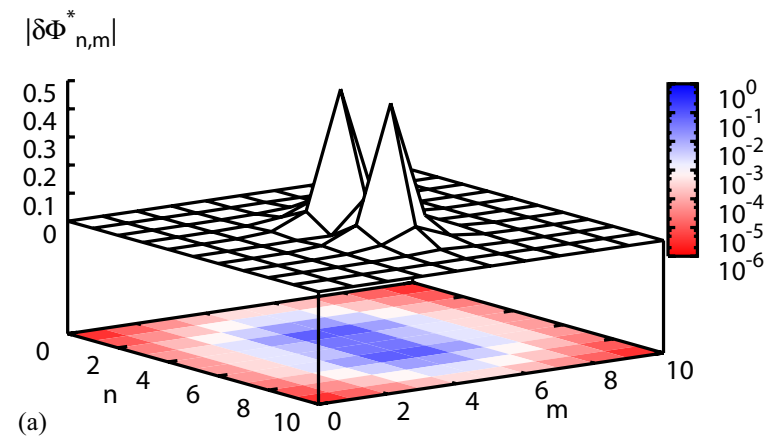

$\left|\delta \Phi^{* *}{ }_{n, m}\right|$

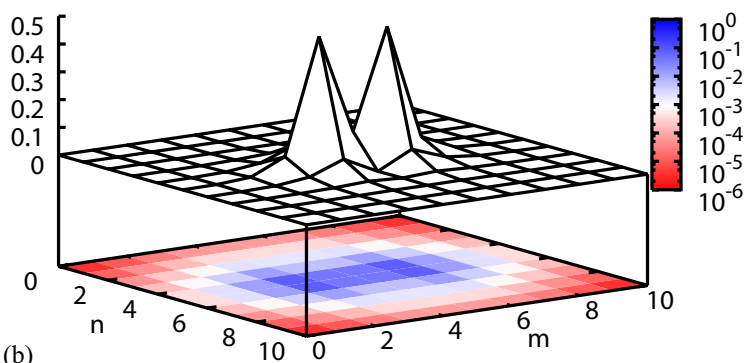

(b)

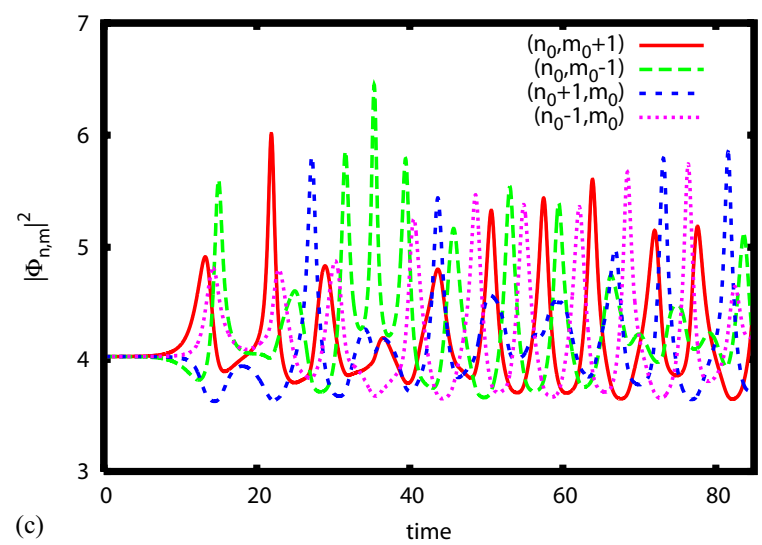

FIG. 8. (Color online) (a) and (b) Intensity profiles of the unstable Floquet eigenvectors, $\delta \Phi^{*} \delta \Phi^{* *}$, corresponding to the bifurcation at $\mu=-0.3$ (for $\alpha=C=1$ ) of the vortex cross with $\omega_{b}=8$, see Fig. 6(d). (c) Time evolution of the lattice field at sites around the center of the same unstable vortex solution. Pulsonic dynamics of the amplitudes is observed, without decay of the vortex pattern.

experienced by out-of-phase bound pairs of solitons in Fig. 4 (for separation $d=1$ ). In any case, a noteworthy numerical finding is that these bifurcations (of bound states and vortex crosses) not only coincide but are also characterized by the divergence of the soliton norm.

\section{B. Vortex squares (with $M=1$ )}

We have also studied the smallest $(M=1)$ vortex squares carrying $S=1$ vorticity. For this purpose, we have performed the continuation of the corresponding solution family, starting from a configuration with nonzero components $\Phi_{n_{0}, m_{0}}$ $=-i \Phi_{n_{0}, m_{0}+1}=-\Phi_{n_{0}+1, m_{0}+1}=i \Phi_{n_{0}+1, m_{0}}=\sqrt{\omega_{b} / 2}$ in the anticontinuum limit, cf. Eq. (6). As in the case of the vortex cross,
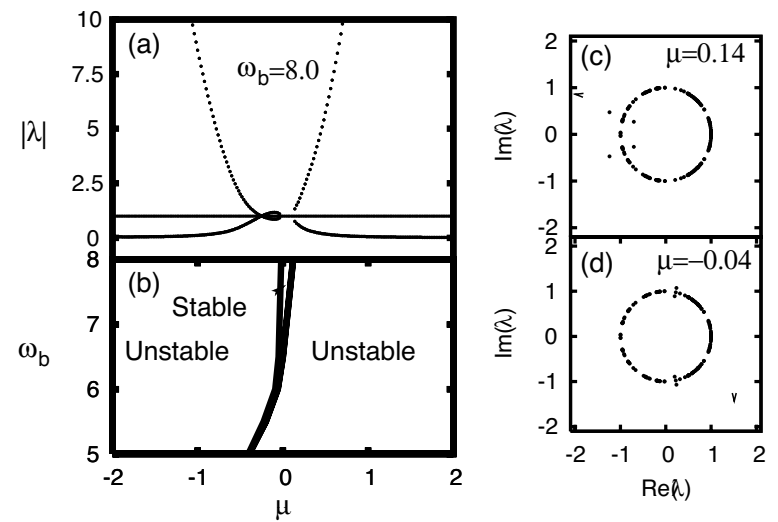

FIG. 9. (a) The absolute value of the Floquet multipliers as a function of $\mu$ for a vortex square of minimum size $(M=1)$ with $\omega_{b}=8$. Two bifurcations are revealed by Floquet distributions in panels (c) and (d). At $\mu=0.14$, we find a Hamiltonian Hopf bifurcation, whereas at $\mu=-0.04$ a quadruplet of eigenvalues leave the unit circle and start a short trip to +1 , from where they leave the unit circle again. The entire stability diagram is represented in panel (b), showing a narrow stability region.

we have first performed the continuation in the coupling constant $\alpha$ to obtain the corresponding solutions for the DNLS model $(\alpha=1, \mu=0)$. Again, for low-frequency vortex squares, we have observed an HHB at some critical value of $\alpha$. For high-frequency solutions, a bifurcation of the same type is observed when the continuation is performed from the DNLS model to values $\mu>0$. In Fig. 9(a), one can observe this bifurcation for the vortex square with $\omega_{b}=8$. The corresponding HHB [see Fig. 9(c)] occurs with a quadruplet of the Floquet eigenvalues leaving the unit circle. The behavior of the unstable solution is the same as for the vortex cross, and, after a transient, a regular soliton with $S=0$ emerges at one of the corner sites of the former vortex square, while the field at three other corners nearly vanishes (i.e., the energy mainly concentrates at a single site of the initial vortex structure).

With the continuation of the vortex square to $\mu<0$, we have again (as in the case of vortex crosses) found that the solutions suffer a destabilizing bifurcation different from that at $\mu>0$. However, the bifurcation for $\mu<0$ [see Fig. 9(d)] is also different from its counterpart for the vortex cross [which was displayed above in Fig. 7(d)]. At some value $\mu<0$, a quadruplet of Floquet multipliers leave the unit circle, to return to it at +1 . After this brief excursion, they immediately leave the unit circle again, and instability grows with $|\mu|$. Unlike its counterpart for the vortex cross, this bifurcation does not correspond to the interchange of stability for the bound state of solitons analyzed in Sec. III C, which actually occurs at a lower value of $\mu$, where the vortex square is already unstable. However, it is remarkable that precisely at this value of $\mu$ the quadruplet of eigenvalues outside the unit circle meet instantaneously at +1 , so that the vortex square is marginally stable at that point.

Profiles of unstable eigenvectors, $\delta \Phi^{*}$ and $\delta \Phi^{* *}$, are shown in Figs. 10(a) and 10(b). Each one is localized at two nonadjacent corners of the plaquette where the vortex square is located. The dynamics triggered by the original solution 

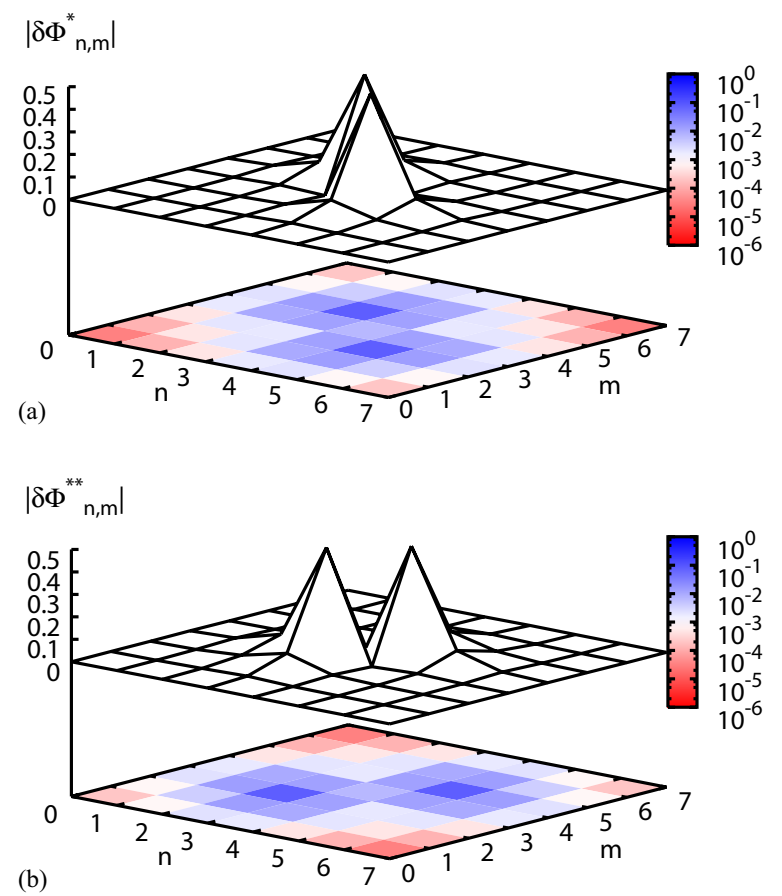

(b)

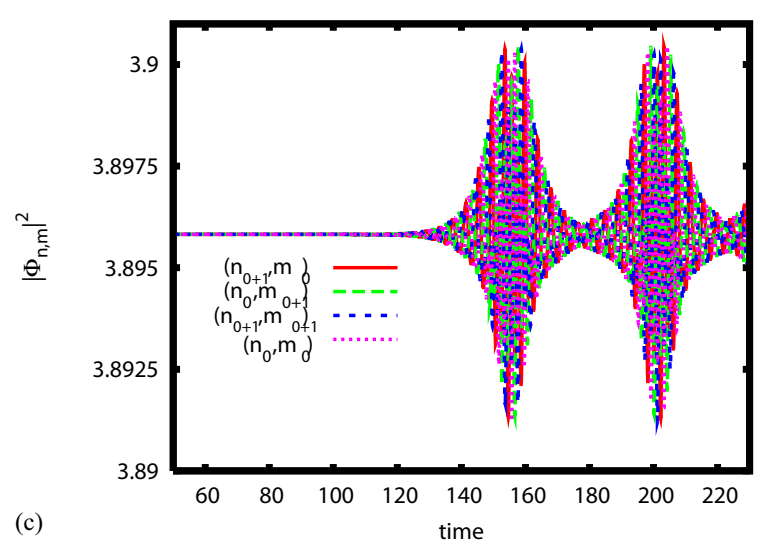

FIG. 10. (Color online) (a) and (b) Intensity profiles of the unstable Floquet eigenvectors, $\delta \Phi^{*}$ and $\delta \Phi^{* *}$, corresponding to the bifurcation, at $\mu=-0.04$ (for $\alpha=C=1$ ), of the vortex square with $\omega_{b}=8$, shown in Fig. 8(d). (c) The time evolution of the lattice field at the vortex-square's corners for the same unstable solution. The simulations reveal periodic evolution of the amplitudes with a clear sequence of energy transfer between the adjacent sites following the same pattern as the current flux in the original vortex solution.

being perturbed by this $\delta \Phi^{*}$ (or equivalently $\delta \Phi^{* *}$ ) is displayed in Fig. 10(c). Again (as in the case of the vortex cross), the vortex pattern is not destroyed (in contrast with the unstable behavior at $\mu>0$ ). Instead, the lattice field at the vortex-square sites develops a periodic pulsonic behavior, in which at least two frequencies can be identified. One of the frequencies accounts for periodic transfer of energy between four corners of the square vortex, following the same path as the flux current: $\left(n_{0}, m_{0}\right) \rightarrow\left(n_{0}, m_{0}+1\right) \rightarrow\left(n_{0}\right.$ $\left.+1, m_{0}+1\right) \rightarrow\left(n_{0}+1, m_{0}\right) \rightarrow\left(n_{0}, m_{0}\right)$. Another noteworthy feature of the dynamics in this case is that the total amount of energy that is periodically transferred between neighboring sites varies, also in a regular periodic fashion, thus giving (a)

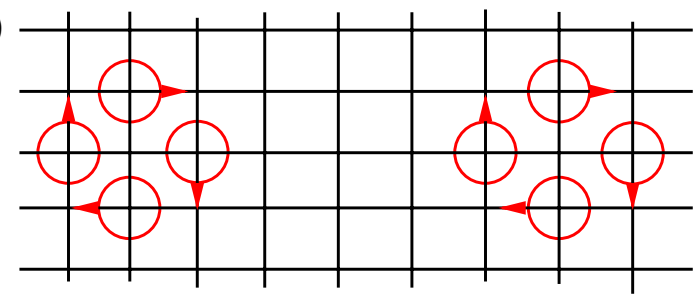

(b)
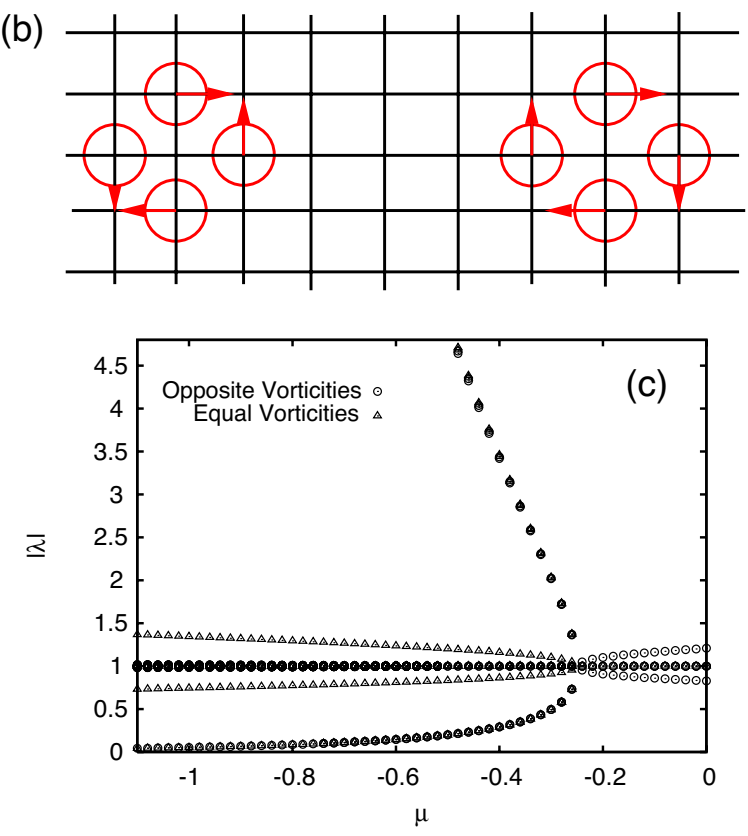

FIG. 11. (Color online) A schematic representation of the inphase (a) and out-of-phase (b) bound states of vortices with $S=1$, in the limit of $\alpha=0, \nu=C=1, \mu=0$. Vectors stand for the instantaneous values of $\Phi_{n, m}$ in the complex plane, with $\left|\Phi_{n, m}\right|=\sqrt{\omega_{b} / 2}$. These solutions are continued in $\alpha$ up to $\alpha=1$, and then continued in $\mu$. Panel (c) shows the evolution of the Floquet multipliers as a function of $\mu$ when $\mu<0$. The results correspond to $\omega_{b}=8$ and the distance between the two vortex centers is set to be $d=5$ [as seen in (a) and (b)].

rise to the second frequency. Again (as happened for the vortex cross), the instability observed at $\mu<0$ induces a pulsonic dynamics of the lattice amplitudes but, in the present case, the dynamics is much more regular. An intriguing numerical observation is that the value of $\mu$ at which the quadruplet of eigenvalues meet at +1 (so that the vortex-square solution momentarily becomes marginally stable) occurs exactly when the soliton norm diverges.

The entire stability diagram for the fundamental vortex squares is presented in Fig. 9(b). Again, we find a narrow stability region for low-frequency vortex squares that expands as the frequency increases.

\section{Bound states of discrete vortex crosses}

As a first step toward the characterization of the stability of more complex 2D arrangements of vortices, we have studied two types of bound states of vortex crosses, with the vortex centers aligned along a lattice axis (say, the $x$ direction). In the two types of bound state, the vortices have equal 
or opposite vorticities, see Figs. 11(a) and 11(b). Both types of solutions were studied on the isotropic lattice $(C=\alpha=\nu$ $=1$ and $\mu<0$ ), and were numerically obtained by the continuation at $\mu=0$ from the anticontinuum limit, followed by the continuation in the direction of negative $\mu$. The Floquet spectrum of the solution was also numerically computed along the continuation path.

At $\mu=0$, bound states of vortices with equal vorticities are stable, while those with opposite vorticities are unstable. To explain this numerical observation, one has to realize that the rightmost member of the soliton set forming the left vortex, and its leftmost counterpart in the right vortex, are out-ofphase (in-phase) in the former (latter) case; see Figs. 11(a) and 11(b). Thus, the stability analysis of bound states of solitons reported above in Sec. III C suggests that the stability of the bound states of vortices is actually dominated by the stability of the local bound state of the two constituent solitons (one from each vortex) that are in closest proximity. This analysis is further validated by comparison of unstable Floquet eigenvalues for the bound state of vortices with opposite vorticities and those for the bound state of in-phase solitons (for the corresponding values of the frequency and separation between the centers).

When $\mu$ decreases, a destabilizing bifurcation occurs, as expected, in the equal-vorticity bound state, precisely at the same value of $\mu$ where the simultaneous instability of the vortex crosses (in Sec. IV A) and the out-of-phase bound state of ordinary solitons occurs. By inspection of the Floquet spectrum for the bound state of vortices, one can clearly identify pairs of eigenvalues associated with each of the instabilities that take place simultaneously at this bifurcation point.

\section{CONCLUSIONS}

In this paper, we have introduced a two-dimensional lattice model with competing on-site self-focusing and intersite self-defocusing cubic nonlinearities (the Salerno model), as a generalization of the recently considered 1D model [10]. The 2D model may find direct application to the description of a dipolar BEC trapped in a deep 2D optical lattice. The general anisotropic version of the 2D model was considered, which made it possible to follow the transition from the 1D lattice to its $2 \mathrm{D}$ counterpart.

Soliton families (of zero vorticity) of two kinds were found: ordinary ones and cuspons, with peakons at the border between the two families. Stability diagrams for the ordinary solitons were produced, and it was found that all cuspons, as well as the peakons, are stable. While the VakhitovKolokolov criterion does not apply to cuspons, it has been verified that it correctly predicts the instability boundary of the ordinary solitons. In direct simulations, unstable solitons turn themselves into localized pulsons. The stability analysis of in-phase and out-of-phase bound states of solitons in the isotropic lattice reveals that there is a stability interchange between both types of bound states, precisely at the same value of the intersite-nonlinearity parameter $(\mu)$ where the soliton norm diverges.

In addition to fundamental solitons, discrete vortices of two types, cross- and square-shaped ones, have also been constructed, and their stability regions identified. In direct simulations, unstable vortices in the 2D Salerno model of the ordinary type transform into regular solitons, while in the model with the competing nonlinearities the instability transforms vortices into localized vortical pulsons, without destroying their topological character. Regarding the stability of bound states of vortex crosses, we have shown that it is determined by the stability of the local bound state of two constituent solitons (forming the two vortices) which are in closest proximity.

The investigation of solitons in the 2D Salerno model, especially in the case of competing nonlinearities, can be continued in various directions. Among relevant problems are the study of more complex bound states of regular solitons and vortices, and the consideration of moving solitons.

\section{ACKNOWLEDGMENTS}

J.G.-G. acknowledges financial support from the MECyD through a FPU grant. This work was partially supported by Spanish DGICyT (Project No. FIS2005-00337), DGA, and BIFI. Work at Los Alamos National Laboratory was supported by the U.S. DOE.
[1] M. Johansson, S. Aubry, Y. B. Gaididei, P. L. Christiansen, and K. Ø. Rasmussen, Physica D 119, 115 (1998); P. G. Kevrekidis, K. Ø. Rasmussen, and A. R. Bishop, Int. J. Mod. Phys. B 15, 2833 (2001).

[2] M. J. Ablowitz and J. F. Ladik, J. Math. Phys. 17, 1011 (1976).

[3] B. A. Malomed and M. I. Weinstein, Phys. Lett. A 220, 91 (1996).

[4] P. G. Kevrekidis, Physica D 183, 68 (2003); B. A. Malomed, J. Fujioka, A. Espinosa-Cerón, R. F. Rodríguez, and S. González, Chaos 16, 013112 (2006).

[5] M. Salerno, Phys. Rev. A 46, 6856 (1992); see also Encyclopedia of Nonlinear Science, edited by A. Scott (Routledge, New York, 2005), p. 819.
[6] D. Cai, A. R. Bishop, and N. Grønbech-Jensen, Phys. Rev. E 53, 4131 (1996); K. Ø. Rasmussen, D. Cai, A. R. Bishop, and N. Grønbech-Jensen, ibid. 55, 6151 (1997).

[7] D. Cai, A. R. Bishop, and N. Grønbech-Jensen, Phys. Rev. E 56, 7246 (1997).

[8] S. V. Dmitriev, P. G. Kevrekidis, B. A. Malomed, and D. J. Frantzeskakis, Phys. Rev. E 68, 056603 (2003).

[9] J. Gómez-Gardeñes, F. Falo, and L. M. Floría, Phys. Lett. A 332, 213 (2004); J. Gómez-Gardeñes, L. M. Floría, M. Peyrard, and A. R. Bishop, Chaos 14, 1130 (2004).

[10] J. Gomez-Gardeñes, B. A. Malomed, L. M. Floría, and A. R. Bishop, Phys. Rev. E 73, 036608 (2006).

[11] K. Goral, K. Rzazewski, and T. Pfau, Phys. Rev. A 61, 
051601(R) (2000); A. Griesmaier, J. Werner, S. Hensler, J. Stuhler, and T. Pfau, Phys. Rev. Lett. 94, 160401 (2005).

[12] Z. D. Li, P. B. He, L. Li, J. Q. Liang, and W. M. Liu, Phys. Rev. A 71, 053611 (2005).

[13] M. G. Vakhitov and A. A. Kolokolov, Izv. Vyssh. Uchebn. Zaved., Radiofiz. 16, 1020 (1973) [Radiophys. Quantum Electr. 16, 783 (1973)]; L. Bergé, Phys. Rep. 303, 260 (1998).

[14] T. Kapitula, P. G. Kevrekidis, and B. A. Malomed, Phys. Rev. E 63, 036604 (2001); D. E. Pelinovsky, P. G. Kevrekidis, and D. J. Frantzeskakis, Physica D, 212, 1 (2005).

[15] B. A. Malomed and P. G. Kevrekidis, Phys. Rev. E 64, 026601 (2001).

[16] E. W. Laedke, K. H. Spatschek, and S. K. Turitsyn, Phys. Rev.
Lett. 73, 1055 (1994).

[17] P. L. Christiansen, Yu. B. Gaididei, K. Ø. Rasmussen, V. K. Mezentsev, and J. J. Rasmussen, Phys. Rev. B 54, 900 (1996); P. L. Christiansen, Yu. B. Gaididei, V. K. Mezentsev, S. L. Musher, K. Ø. Rasmussen, J. J. Rasmussen, I. V. Ryzhenkova, and S. K. Turitsyn, Phys. Scr. 67, 160 (1996).

[18] J. Gómez-Gardeñes, L. M. Floría, and A. R. Bishop, Physica D 216, 31 (2006).

[19] P. G. Kevrekidis, D. J. Frantzeskakis, R. Carretero-Gonzalez, B. A. Malomed, and A. R. Bishop, Phys. Rev. E 72, 046613 (2005).

[20] J.-C. van der Meer, Nonlinearity 3, 1041 (1990). 